\title{
A Quantum Version of the Désarménien Matrix
}

\author{
ANNA STOKKE \\ a.stokke@uwinnipeg.ca \\ Department of Mathematics and Statistics, University of Winnipeg, 515 Portage Avenue, Winnipeg, Manitoba,
} Canada R3B $2 E 9$

Received May 14, 2004; Revised March 9, 2005; Accepted April 15, 2005

Abstract. We use elements in the quantum hyperalgebra to define a quantum version of the Désarménien matrix. We prove that our matrix is upper triangular with ones on the diagonal and that, as in the classical case, it gives a quantum straightening algorithm for quantum bideterminants. We use our matrix to give a new proof of the standard basis theorem for the $q$-Weyl module. As well, we show that the standard basis for the $q$-Weyl module and the basis dual to the standard basis for the $q$-Schur module are related by the quantum Désarménien matrix.

Keywords: $\quad q$-Weyl module, $q$-Schur module, Désarménien matrix, quantum straightening algorithm, standard basis theorem

\section{Introduction}

Let $K$ be an infinite field, $n$ and $r$ fixed positive integers, and $\lambda$ a fixed partition of $r$. In the classical case, the Désarménien matrix corresponding to $\lambda$ and $n$ is an upper triangular unimodular matrix that plays an interesting role in the representation theory of the general linear group $G L(n, K)$. It was first introduced in [4] where it was defined using Capelli operators which, although it is not pointed out there, are elements in the hyperalgebra for $G L(n, K)$. Its original purpose was to act as tool for writing a given bideterminant as a linear combination of bideterminants which come from semistandard $\lambda$-tableaux. In [3], Désarménien showed that the entries in the matrix may be determined in a combinatorial manner by examining pairs of semistandard $\lambda$-tableaux with entries from the set $\{1, \ldots, n\}$.

Since its introduction, the Désarménien matrix has appeared in a number of places relating to the polynomial representations of $G L(n, K)$. J. A. Green gives a very nice proof of the Carter-Lusztig basis theorem (see [1]) for the Weyl module $\Delta(\lambda)$ for $G L(n, K)$ in [7]. At the heart of his proof is the Désarménien matrix. The proof directly shows that the Désarménien matrix is the change of basis matrix between the Carter-Lusztig basis for $\Delta(\lambda)$ and the basis dual to the standard basis for the Schur module. In [6], J. A. Green uses a version of the Désarménien matrix to give a basis of codeterminants for the Schur algebra $S(n, r)$.

A symplectic version of the Désarménien matrix is given in [13]. This matrix has many of the properties of the original Désarménien matrix and a version of it has proved useful in providing bases of symplectic codeterminants for the symplectic Schur algebras in [2].

In Section 4 we will define quantum versions of the Capelli operators and use them to define a quantum Désarménien matrix. We prove that our matrix is upper triangular with ones on the diagonal. As well, we show that it can be used to give a quantum straightening 
algorithm for quantum bideterminants. Another quantum straightening algorithm is given in [11].

A quantum version of the Carter-Lusztig standard basis theorem for the $q$-Weyl module corresponding to $\lambda$ was proved by R. M. Green in [8]. The contravariant dual to the $q$-Weyl module, called the $q$-Schur module, has a basis of bideterminants given by semistandard $\lambda$ tableaux so the $q$-Weyl module also has a basis which is dual to this basis. Our Désarménien matrix turns out to be the connection between these two bases. We prove this by giving a new proof of the standard basis theorem from which this fact emerges.

\section{Young tableaux}

Throughout the article, $K$ shall be an infinite field, $n$ and $r$ shall be fixed positive integers, and $\lambda$ shall be a fixed partition of $r$.

A $k$-tuple $\lambda=\left(\lambda_{1}, \lambda_{2}, \ldots, \lambda_{k}\right)$ of positive integers is a partition of $r$ if $\lambda_{1} \geq \lambda_{2} \geq \cdots \geq$ $\lambda_{k}$ and $\sum_{i=1}^{k} \lambda_{i}=r$. The Young diagram of shape $\lambda$ is a collection of $r$ boxes arranged in $k$ left justified rows with the $i$ th row consisting of $\lambda_{i}$ boxes. A $\lambda$-tableau is obtained by filling the Young diagram of shape $\lambda$ with entries from the set $\{1,2, \ldots, n\}$. A $\lambda$-tableau is called semistandard if the entries in its rows are weakly increasing from left to right and strictly increasing from top to bottom.

Let $I(n, r)$ denote the set of $r$-tuples with entries from the set $\{1,2, \ldots, n\}$. It is often convenient to associate to a given $\lambda$-tableau a sequence $I=\left(i_{1}, i_{2}, \ldots, i_{r}\right)$ in $I(n, r)$ and to associate to a sequence $I$ in $I(n, r)$ a $\lambda$-tableau $T_{I}$. We do this by letting $T_{I}$ denote the $\lambda$-tableau obtained by filling the Young diagram of shape $\lambda$ canonically down the columns with the entries in $I$ working from left to right and from top to bottom.

Example 2.1 Let $\lambda=(3,2)$. Then $T=$\begin{tabular}{|l|l|l|l|l|l|l|l}
1 & 2 & 3 \\
4 & 6 & is a semistandard $\lambda$-tableau. We may
\end{tabular} write $T=T_{I}$ where $I=(1,4,2,6,3)$.

We will often make use of the basic tableau, which we denote by $T_{\lambda}$. If $s$ is the number of rows in the Young diagram of shape $\lambda$, then $T_{\lambda}$ is the $\lambda$-tableau in which every entry in the $i$ th row is equal to $i$, for $1 \leq i \leq s$. We denote by $J(\lambda)$ the sequence in $I(n, r)$ that satisfies $T_{\lambda}=T_{J(\lambda)}$.

Example 2.2 If $\lambda=(3,2)$, then $T_{\lambda}=$\begin{tabular}{|l|l|l}
\hline & 1 & 1 \\
2 & 2
\end{tabular}$\quad$ and $J(\lambda)=(1,2,1,2,1)$.

The symmetric group $S_{r}$ acts on $I(n, r)$ on the right by

$$
I \cdot \sigma=\left(i_{1 \sigma}, \ldots, i_{r \sigma}\right), \quad I=\left(i_{1}, i_{2}, \ldots, i_{r}\right) \in I(n, r) .
$$

This action induces an action of $S_{r}$ on the set of $\lambda$-tableaux with entries from $\{1,2, \ldots, n\}$ by $T_{I} \cdot \sigma=T_{I \cdot \sigma}$. Let $I=(1,2, \ldots, r)$. We denote the tableau $T_{I}$, for this particular choice of $I$, by $\hat{T}_{\lambda}$. The column stabilizer of $\hat{T}_{\lambda}$, denoted $C\left(\hat{T}_{\lambda}\right)$, is the set of $\sigma \in S_{r}$ which preserve the columns of $\hat{T}_{\lambda}$ under this action. 


\section{The quantum hyperalgebra}

In this section, we construct the quantum analogue of the hyperalgebra for $G L(n, K)$. We then discuss the quantum analogues of the Schur and Weyl modules.

Let $q$ be an indeterminate and let $\mathcal{A}=\mathbb{Z}\left[q, q^{-1}\right]$ denote the ring of Laurent polynomials in $q$. Let $\mathbb{Q}(q)$ be the field of quotients of $\mathcal{A}$. The quantum enveloping algebra, which we denote by $U_{\mathbb{Q}(q)}$, is the associative $\mathbb{Q}(q)$-algebra with generators $E_{i}, F_{i}, K_{j}, K_{j}^{-1}$ with $1 \leq i<n, 1 \leq j \leq n$, subject to the relations that follow. We let $K_{i, i+1}$ denote $K_{i} K_{i+1}^{-1}$.

$$
\begin{array}{ll}
K_{i} K_{i}^{-1}=K_{i}^{-1} K_{i}=1 & K_{i} K_{j}=K_{j} K_{i} \\
K_{i} E_{j}=q^{\delta_{i, j}-\delta_{i, j+1}} E_{j} K_{i} & K_{i} F_{j}=q^{\delta_{i, j+1}-\delta_{i j}} F_{j} K_{i} \\
E_{i} E_{j}=E_{j} E_{i} \quad \text { if }|i-j|>1 & F_{i} F_{j}=F_{j} F_{i} \quad \text { if }|i-j|>1 \\
E_{i} F_{j}-F_{j} E_{i}=\delta_{i j} \frac{K_{i, i+1}-K_{i, i+1}^{-1}}{q-q^{-1}} & \\
E_{i}^{2} E_{j}-\left(q+q^{-1}\right) E_{i} E_{j} E_{i}+E_{j} E_{i}^{2}=0 & \\
F_{i}^{2} F_{j}-\left(q+q^{-1}\right) F_{i} F_{j} F_{i}+F_{j} F_{i}^{2}=0 & \text { if }|i-j|=1 \\
\end{array}
$$

The algebra $U_{\mathbb{Q}(q)}$ is a Hopf algebra over $\mathbb{Q}(q)$ with comultiplication

$$
\Delta: U_{\mathbb{Q}(q)} \rightarrow U_{\mathbb{Q}(q)} \otimes U_{\mathbb{Q}(q)}
$$

defined by

$$
\Delta\left(E_{i}\right)=1 \otimes E_{i}+E_{i} \otimes K_{i, i+1}, \quad \Delta\left(F_{i}\right)=K_{i, i+1}^{-1} \otimes F_{i}+F_{i} \otimes 1, \quad \Delta\left(K_{i}\right)=K_{i} \otimes K_{i} .
$$

Note that $U_{\mathbb{Q}(q)}$ possesses other comultiplications which are preferred by some authors.

Let $a$ be a nonnegative integer. Define

$$
[a]=\frac{q^{a}-q^{-a}}{q-q^{-1}}
$$

and quantum factorial as

$$
[a] !=[a][a-1] \ldots[1] .
$$

For $X \in U_{\mathbb{Q}(q)}$ and $a \in \mathbb{N}$, let

$$
X^{(a)}=\frac{X^{a}}{[a] !} \quad \text { and }\left(\begin{array}{c}
K_{j} \\
a
\end{array}\right)=\prod_{s=1}^{a} \frac{q^{-s+1} K_{j}-q^{s-1} K_{j}^{-1}}{q^{s}-q^{-s}}
$$


The integral form $U_{\mathcal{A}}$ of $U_{\mathbb{Q}(q)}$, given in [12], is a quantum version of Kostant's $\mathbb{Z}$-form. It is the Hopf $\mathcal{A}$-subalgebra of $U_{\mathbb{Q}(q)}$ generated by the elements

$$
E_{i}^{(a)}, F_{i}^{(a)}, K_{j}, K_{j}^{-1},\left(\begin{array}{c}
K_{j} \\
a
\end{array}\right), \quad a \in \mathbb{N}, \quad 1 \leq i<n, 1 \leq j \leq n .
$$

Let $t$ be a unit in $K$ such that $t^{2} \neq 1$. We may regard our field $K$ as an $\mathcal{A}$-algebra by letting $q \in \mathcal{A}$ act on $K$ by multiplication by $t \in K$. The quantum hyperalgebra is defined as

$$
U_{K}=U_{\mathcal{A}} \otimes_{\mathcal{A}} K
$$

We will often say that $M$ is a $U_{K}$-module, meaning that $M$ is a $U_{\mathcal{A}}$-module so we have a $U_{K}$-module via base change. We write $u \in U_{K}$ to mean the image of $u$ in $U_{K}$ via the map $\phi: U_{\mathcal{A}} \rightarrow U_{K}$ defined by $\phi(u)=u \otimes 1$.

Let $V_{\mathbb{Q}(q)}$ be the $n$-dimensional vector space over $\mathbb{Q}(q)$ with basis $v_{1}, \ldots, v_{n}$. Then $V_{\mathbb{Q}(q)}$ is a $U_{\mathbb{Q}(q)}$-module, called the natural module, via

$$
E_{i} v_{j}=\delta_{i+1, j} v_{i}, \quad F_{i} v_{j}=\delta_{i j} v_{i+1}, \quad K_{i} v_{j}=q^{\delta_{i j}} v_{j}, \quad K_{i}^{-1} v_{j}=q^{-\delta_{i j}} v_{j} .
$$

Denote the $U_{\mathcal{A}}$-submodule of $V_{\mathbb{Q}(q)}$ generated by $v_{1}, \ldots, v_{n}$ by $V_{\mathcal{A}}$. The coassociative comultiplication $\Delta$ defined in (3.1) defines a $U_{\mathcal{A}}$-module action on $V_{\mathcal{A}}^{\otimes r}$. We will let $V^{\otimes r}$ denote the $U_{K}$-module obtained by base change. Given $I=\left(i_{1}, i_{2}, \ldots, i_{r}\right) \in I(n, r)$, define $v_{I} \in V_{\mathcal{A}}$ by $v_{I}=v_{i_{1}} \otimes v_{i_{2}} \otimes \cdots \otimes v_{i_{r}}$.

Define $z_{\lambda} \in V_{\mathcal{A}}^{\otimes r}$ by

$$
z_{\lambda}=\sum_{\sigma \in C\left(\hat{T}_{\lambda}\right)}(-q)^{-l(\sigma)} v_{J(\lambda) \cdot \sigma}
$$

where $l(\sigma)$ denotes the length of the permutation $\sigma \in S_{r}$ and $J(\lambda)$ and $C\left(\hat{T}_{\lambda}\right)$ are defined in Section 2. Let $\Delta(\lambda)_{\mathcal{A}}$ denote the left $U_{\mathcal{A}}$-submodule of $V_{\mathcal{A}}^{\otimes r}$ generated by $z_{\lambda}$. The $q$-Weyl module is the left $U_{K}$-module defined by

$$
\Delta_{q}(\lambda)=\Delta(\lambda)_{\mathcal{A}} \otimes_{\mathcal{A}} K
$$

We now define a quantum version of the algebra of polynomial functions on the set of $n \times n$ matrices over $K$. As in [15], an $n \times n$ matrix $A=\left(a_{i j}\right)_{1 \leq i, j \leq n}$ with entries in an $\mathcal{A}$-algebra is a $q$-matrix if its entries satisfy the following relations:

$$
\begin{array}{ll}
a_{i k} a_{i l}=q^{-1} a_{i l} a_{i k} & 1 \leq k<l \leq n \\
a_{i k} a_{j k}=q^{-1} a_{j k} a_{i k} & 1 \leq i<j \leq n \\
a_{i l} a_{j k}=a_{j k} a_{i l} & 1 \leq i<j \leq n, \quad 1 \leq k<l \leq n \\
a_{i k} a_{j l}-a_{j l} a_{i k}=\left(q^{-1}-q\right) a_{i l} a_{j k} & 1 \leq i<j \leq n, \quad 1 \leq k<l \leq n .
\end{array}
$$


Define $A_{q}(n)$ to be the $\mathcal{A}$-algebra with generators $x_{i j}$ subject to the relations which require $X=\left(x_{i j}\right)_{1 \leq i, j \leq n}$ to be a $q$-matrix.

It can be checked (see [15]) that $A_{q}(n)$ is a $U_{\mathcal{A}}$-module with action

$$
E_{i} x_{k l}=\delta_{i+1, l} x_{k i}, \quad F_{i} x_{k l}=\delta_{i l} x_{k, i+1}, \quad K_{i} x_{k l}=q^{\delta_{i l}} x_{k l}, \quad K_{i}^{-1} x_{k l}=q^{-\delta_{i l}} x_{k l}
$$

and $E_{i}(P Q)=P\left(E_{i} Q\right)+\left(E_{i} P\right)\left(K_{i, i+1} Q\right), F_{i}(P Q)=\left(K_{i, i+1}^{-1} P\right)\left(F_{i} Q\right)+\left(F_{i} P\right) Q, K_{i}(P Q)$ $=\left(K_{i} P\right)\left(K_{i} Q\right)$ where $P, Q \in A_{q}(n)$. Of course we get a $U_{K}$-module by base change and we also denote this module by $A_{q}(n)$.

If $I=\left(i_{1}, \ldots, i_{r}\right)$ and $J=\left(j_{1}, \ldots, j_{r}\right)$ belong to $I(n, r)$ with $i_{1}<i_{2}<\cdots<i_{r}$, define $\operatorname{det}_{q} X_{J}^{I}$ in $A_{q}(n)$ by

$$
\operatorname{det}_{q} X_{J}^{I}= \begin{cases}\sum_{\sigma \in S_{r}}(-q)^{-\ell(\sigma)} x_{i_{1} j_{\sigma(1)}} x_{i_{2} j_{\sigma(2)}} \ldots x_{i_{r} j_{\sigma(r)}} & \text { if } j_{1}<j_{2}<\cdots<j_{r} \\ \sum_{\sigma \in S_{r}}(-q)^{-\ell(\sigma)} x_{i_{\sigma^{-1}(1)} j_{1}} x_{\sigma^{-1}(2) j_{2}} \ldots x_{i_{\sigma^{-1}(r)} j_{r}} & \text { otherwise. }\end{cases}
$$

Given $\lambda$-tableaux $S$ and $T$, where $S$ is column increasing, the quantum bideterminant $(S: T) \in A_{q}(n)$ is defined as

$$
(S: T)=\left(\operatorname{det}_{q} X_{T(1)}^{S(1)}\right)\left(\operatorname{det}_{q} X_{T(2)}^{S(2)}\right) \cdots\left(\operatorname{det}_{q} X_{T(s)}^{S(s)}\right)
$$

where $s$ is the number of columns in the Young diagram of shape $\lambda$ and $T(i)$ (respectively $S(i)$ ) denotes the subsequence corresponding to the entries in the $i$ th column of $T$ (respectively $S$ ). In this article, we are only interested in bideterminants of the form $\left(T_{\lambda}: T\right)$, where $T_{\lambda}$ is the basic $\lambda$-tableau defined in Section 2 . We take $[T]$ to represent the bideterminant $\left(T_{\lambda}: T\right)$. Note that $\lambda$ is fixed throughout, so $T$ is always a $\lambda$-tableau.

Let $\nabla(\lambda)_{\mathcal{A}}$ be the $\mathcal{A}$-span of the quantum bideterminants $[T]$, where the set runs over all $\lambda$-tableaux $T$ with entries from the set $\{1, \ldots, n\}$. The fact that $\nabla(\lambda)_{\mathcal{A}}$ is a $U_{\mathcal{A}}$-invariant submodule of $A_{q}(n)$ follows from [14, Proposition 2.1]. The $q$-Schur module, denoted $\nabla_{q}(\lambda)$ is the $U_{K}$-module given by base change.

There is an antiautormorphism $\tau: U_{\mathcal{A}} \rightarrow U_{\mathcal{A}}$ defined by

$$
\tau\left(E_{i}\right)=F_{i}, \quad \tau\left(F_{i}\right)=E_{i}, \quad \tau\left(K_{i}\right)=K_{i} .
$$

We denote the induced map on $U_{K}$ by $\tau$ as well.

If $M$ is a $U_{K}$-module, the contravariant dual of $M$, denoted $M^{\circ}$, is just the dual space $M^{*}$ with action defined as

$$
u f(m)=f(\tau(u) m), \quad u \in U_{K}, \quad m \in M, \quad f \in M^{*} .
$$

In Section 5, we define a contravariant form on $V^{\otimes r}$ and use it to explicitly construct the contravariant dual to $\nabla_{q}(\lambda)$. The resulting module will be isomorphic to $\Delta_{q}(\lambda)$. For a proof of the following, see [5, Proposition 4.1.6]. 
Theorem 3.1 The $q$-Weyl module is the contravariant dual to $\nabla_{q}(\lambda)$; that is

$$
\left(\nabla_{q}(\lambda)\right)^{\circ} \cong \Delta_{q}(\lambda)
$$

We shall frequently use the following standard basis theorem for $\nabla_{q}(\lambda)$. There are several different proofs of this result; see for instance [9] or [10].

Theorem 3.2 The set $\{[T]: T$ is a semistandard $\lambda$-tableau $\}$ forms a $K$-basis for $\nabla_{q}(\lambda)$.

\section{A quantum version of the Désarménien matrix}

In this section we define a quantum version of the Désarménien matrix. We introduce a number of technical lemmas which will ease our task. Using (3.4), we may determine the action of $E_{i}, F_{i}$ or $K_{i}$ on a bideterminant [T]. For a discussion of this action on bideterminants, see [11, p. 7 and Example 2.2].

To define quantum analogues of the Capelli operators, we first define $E_{i j}$ and $F_{i j}$ recursively in $U_{K}$ as follows:

$$
E_{i}=E_{i, i+1}, E_{i j}=E_{i} E_{i+1, j}-q^{-1} E_{i+1, j} E_{i} \quad \text { and } F_{i j}=F_{i+1, j} F_{i}-q^{-1} F_{i} F_{i+1, j}
$$

It can be quite difficult to compute $E_{i j}[T]$ for an arbitrary $\lambda$-tableau $T$. We will frequently be encountering tableaux for which the task becomes simplified, as illustrated by the following example and Lemma 4.2.

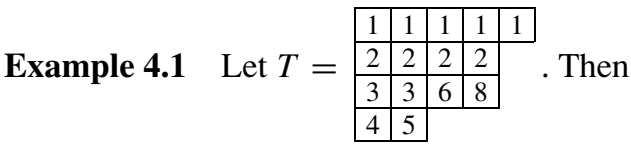

$$
\begin{aligned}
E_{36}[T] & =\left(E_{3} E_{46}-q^{-1} E_{46} E_{3}\right)[T] \\
& =E_{3} E_{46}[T], \text { since } E_{3}[T]=0(\text { see }[11, \text { Example 2.2] }) \\
& =E_{3}\left(E_{4} E_{5}-q^{-1} E_{5} E_{4}\right)[T] \\
& =E_{3} E_{4} E_{5}[T] \text { since } E_{3} E_{5} E_{4}[T]=0 .
\end{aligned}
$$

Lemma 4.2 Suppose that $T$ is a column increasing $\lambda$-tableau that coincides with $T_{\lambda}$ in the first $i-1$ rows. Suppose also that if $m$ is an integer with $i<m<j$, then $m$ does not appear in the ith row of $T$. Then $E_{i j}[T]=E_{i} E_{i+1} \ldots E_{j-1}[T]$.

Proof: We fix $j$ and induct on $i$ in the operator $E_{i j}$. If $i=j-1$, the result is trivial. Suppose that $E_{k j}[T]=E_{k} E_{k+1} \ldots E_{j-1}[T]$ for $i<k \leq j-1$. If an $i+1$ appears in $T$, our hypotheses force an $i$ to appear in a column above it so $E_{i}[T]=0$. Thus $E_{i j}[T]=E_{i} E_{i+1, j}[T]-q^{-1} E_{i+1, j} E_{i}[T]=E_{i} E_{i+1} \ldots E_{j-1}[T]$. 
Given $I \in I(n, r)$, define $d(I)$ to be the number of pairs $(a, b)$ which satisfy $a<b$ and $i_{a}<i_{b}$. For instance, if $I=(1,2,1,4,3)$, then $d(I)=7$.

Lemma 4.3 Suppose that $j>i$. Then $E_{i j}^{r}\left(x_{1 j}^{r}\right)=[r] ! x_{1 i}^{r}$.

Proof: We will show that $E_{i j}^{s}[T]=[s] ! \sum_{I} q^{-d(I)}\left[T_{I}\right]$ for $1 \leq s \leq r$ where the sum runs over all $r$-tuples $I$ which contain $s$ entries equal to $i$ and $r-s$ entries equal to $j$ and $T$ is the one-row tableau with $r$ entries equal to $j$. Then, if we let $s=r$, this is precisely the statement of the Lemma. The statement holds for $s=1$ since

$$
E_{i j}[T]=E_{i} E_{i+1} \ldots E_{j-1}[T]=E_{i} E_{i+1} \ldots E_{j-2} \sum_{M} q^{-d(M)}\left[T_{M}\right]=\sum_{J} q^{-d(J)}\left[T_{J}\right],
$$

where the sum runs over the $r$-tuples $J$ which contain one $i$ and $r-1$ entries equal to $j$.

Now suppose that for all $1 \leq m \leq s$ we have $E_{i j}^{m}[T]=[m] ! \sum_{I} q^{-d(I)}\left[T_{I}\right]$ where the sum runs over all $r$-tuples $I$ which contain $m$ entries equal to $i$ and $r-m$ entries equal to $j$. Then $E_{i j}^{s}[T]=[s-1] ! \sum_{I} q^{-d(I)} E_{i j}\left[T_{I}\right]$ where the sum is over the $r$-tuples $I$ which contain $s-1$ entries equal to $i$ and $r-(s-1)$ entries equal to $j$. Now $\sum_{I} E_{i j}\left[T_{I}\right]=\sum_{J} \gamma_{I J}\left[T_{J}\right]$ where $\gamma_{I J} \in \mathbb{Z}\left[q, q^{-1}\right]$ and the sum runs over all $J$ which contain $s$ entries equal to $i$ and $r-s$ entries equal to $j$. Furthermore, $\gamma_{I J}=0$ in case $J$ cannot be formed by changing a $j$ in $I$ to an $i$. Thus $E_{i j}^{s}[T]=[s-1] ! \sum_{J} \sum_{I} q^{-d(I)} \gamma_{I J}\left[T_{J}\right]$, where the sum runs over all $J$ which contain $s$ entries equal to $i$ and $r-s$ entries equal to $j$ and all $I$ which contain $s-1$ entries equal to $i$ and $r-(s-1)$ entries equal to $j$. We now prove that for each $J$ with $s$ entries equal to $i$ and $r-s$ entries equal to $j$, we have $\sum_{I} q^{-d(I)} \gamma_{I J}=[s] q^{-d(J)}$, where the sum is over all $I$ which yield $J$ when a $j$ in $I$ is changed to an $i$. Fix such a $J$. Let $I_{1}$ be the $r$-tuple that comes from changing the first $i$ from the right in $J$ to a $j, I_{2}$ the $r$-tuple that comes from changing the second $i$ from the right in $J$ to a $j$, etc. Then $\sum_{I} q^{-d(I)} \gamma_{I J}=\sum_{k=1}^{s} q^{-d\left(I_{k}\right)} \gamma_{I_{k} J}$.

For each $k$ with $1 \leq k \leq s$, let $\alpha_{k}$ be the number of $j$ 's to the right of the $k$ th $i$ from the right in $J$. Then $d\left(I_{k}\right)=\sum_{i=1}^{s} \alpha_{i}-\alpha_{k}+(s-k)=d(J)-\alpha_{k}+(s-k)$. To examine $\gamma_{I_{k} J}$ we apply $E_{i} E_{i+1} \ldots E_{j-1}$ to $\left[T_{I_{k}}\right]$ and look at the coefficient of the portion of this sum that gives $J$. Since $q^{-\alpha_{j}}$ is introduced by the $E_{j-1}$ operator and $q^{k-1}$ is introduced by the $E_{i}$ operator, $\gamma_{I_{k} J}=q^{-\alpha_{k}} q^{k-1}$.

It follows that

$$
\sum_{k=1}^{s} q^{-d\left(I_{k}\right)} \gamma_{I_{k} J}=\sum_{k=1}^{s} q^{-d(J)} q^{-s+2 k-1}=\sum_{k=1}^{s} q^{-d(J)}[s]
$$

and this completes the proof.

Lemma 4.4 Let $T$ be a semistandard $\lambda$-tableau that coincides with $T_{\lambda}$ in the first $i-1$ rows. Suppose that there are $r$ entries equal to $j$ in the ith row of $T$. Suppose also that if $m$ is an integer with $i<m<j$ then $m$ does not occur in the ith row of $T$. Then $E_{i j}^{r}[T]=[r] ![S]$ 
where $S$ is identical to $T$ except that the $r$ entries equal to $j$ in the ith row of $T$ have been replaced by $i$ 's.

Proof: Suppose that the $r$ entries equal to $j$ appear in the $i$ th row of $T$ in columns $a$ through $b=a+r$. We argue that applying $E_{i j}^{r}$ affects only the portion of the tableau in which these $j$ 's occur. The lemma then follows from Lemma 4.3.

From our assumptions on $T$, it follows that if $k>b$ there are no entries between $i-1$ and $j+1$ in the $k$ th column $T(k)$ of $T$. Thus, the operators $E_{i}, \ldots, E_{j-1}$ do not affect these columns and $K_{\ell, \ell+1}^{-1}[T(k)]=[T(k)]$ for $k>b$ and $i \leq \ell \leq j-1$.

If $i<m<j$ and $m$ appears in $T$, it must appear below the $i$ th row in some column $T(k)$ with $k<a$. Suppose that $[S]$ is a bideterminant in the sum $E_{j-1}[T]$ that comes from changing a $j$ below the $i$ th row of $T$ to a $j-1$. Any bideterminant in the sum $E_{i}\left(E_{i+1} \ldots E_{j-2}[S]\right)$ comes from changing an $i+1$ below the $i$ th row to an $i$ in some bideterminant $\left[S^{\prime}\right]$ in the sum $E_{i+1} \ldots E_{j-1}[S]$. There is an $i$ in the $i$ th row above this $i$, so $E_{i}\left[S^{\prime}\right]=0$ and $E_{i}\left(E_{i+1} \ldots E_{j-2}[S]\right)=0$. Thus all bideterminants in the sum $E_{j-1}[T]$ come from changing a $j$ in the $i$ th row of $T$ to a $j-1$. Continuing this argument, we see that every bideterminant in the sum $E_{i} E_{i+1} \ldots E_{j-1}[T]$ comes from changing a $j$ in the $i$ th row to an $i$. Inductively, it follows that $E_{i j}^{r}$ affects only those $j$ 's in the $i$ th row of $T$.

We now define operators $E_{T}$ and $F_{T}$ in $U_{K}$. The operators $F_{T}$ also appear in [8]. Given a semistandard $\lambda$-tableau $T$ with $k \leq n$ rows, define

$$
E_{T}=\prod_{\substack{1 \leq i \leq k \\ i<j \leq n}} E_{i j}^{\left(\gamma_{i j}\right)} \text { and } F_{T}=\prod_{\substack{1 \leq i \leq k \\ i<j \leq n}} F_{i j}^{\left(\gamma_{i j}\right)}
$$

where $\gamma_{i j}$ is the number of entries equal to $j$ in row $i$ of $T$. The product $E_{T}$ is ordered

$$
\begin{aligned}
E_{T} & =E_{k n}^{\left(\gamma_{k n}\right)} \ldots E_{k, k+1}^{\left(\gamma_{k, k+1}\right)} \ldots E_{2 n}^{\left(\gamma_{2 n}\right)} \ldots E_{23}^{\left(\gamma_{23}\right)} E_{1 n}^{\left(\gamma_{1 n}\right)} \ldots E_{13}^{\left(\gamma_{13}\right)} E_{12}^{\left(\gamma_{12}\right)} \text { and } \\
F_{T} & =F_{12}^{\left(\gamma_{12}\right)} F_{13}^{\left(\gamma_{13}\right)} \ldots F_{1 n}^{\left(\gamma_{1 n}\right)} F_{23}^{\left(\gamma_{23}\right)} \ldots F_{2 n}^{\left(\gamma_{2 n}\right)} \ldots F_{k, k+1}^{\left(\gamma_{k, k+1}\right)} \ldots F_{k n}^{\left(\gamma_{k n}\right)} .
\end{aligned}
$$

Definition 4.5 Given a semistandard $\lambda$-tableau $T$ and a column increasing $\lambda$-tableau $S$, define $\Omega_{q}(S, T)=c$ where $c$ is the coefficient of $\left[T_{\lambda}\right]$ in the expansion of $E_{T}[S]$ into a linear combination of basis elements.

Order the set of $\lambda$-tableaux by declaring $S<T$ if the row sequence of $S$ is less than the row sequence of $T$ with respect to the lexicographic order on $I(n, r)$. In Example 4, for instance, $T_{1}<T_{2}$.

Definition 4.6 The quantum Désarménien matrix is the matrix

$$
\Omega_{q}=\left[\Omega_{q}\left(T_{I}, T_{J}\right)\right]_{I, J \in \mathcal{I}_{\lambda}}
$$

where $\mathcal{I}_{\lambda}=\left\{I \in I(n, r): T_{I}\right.$ is a semistandard $\lambda$-tableau $\}$. 
We begin with an example to motivate the next theorem.

Example 4.7 Let $T=$\begin{tabular}{|l|l|l|}
\hline 1 & 1 & 6
\end{tabular} . Then $E_{T}[T]=E_{25}^{(2)} E_{16}[T]=\left[\begin{array}{l|l|l}\hline 1 & 1 & 1\end{array}\right]$ so $\Omega_{q}(T, T)$ $=1$.

Theorem 4.8 Suppose that $T$ is a semistandard $\lambda$-tableau. Then $\Omega_{q}(T, T)=1$.

Proof: By Lemma 4.4, we have $E_{12}^{\gamma_{12}}[T]=\left[\gamma_{12}\right]$ ! $[U]$ where $U$ is the single tableau that results from changing the $\gamma_{12}$ entries equal to 2 in the first row of $T$ to ones. Thus, $E_{12}^{\left(\gamma_{12}\right)}[T]=$ $[U]$. Suppose that $E_{1, j-1}^{\left(\gamma_{1, j-1}\right)} \ldots E_{12}^{\left(\gamma_{12}\right)}[T]=[U]$ where the first $\gamma_{11}+\gamma_{12}+\cdots+\gamma_{1, j-1}$ columns of the first row of $U$ contain ones while the remainder of $U$ is identical to $T$. By Lemma 4.4, we have $E_{1 j}^{\left(\gamma_{1 j}\right)}[U]=\left[U^{\prime}\right]$, where the entries in the first $\gamma_{11}+\gamma_{12}+\cdots+\gamma_{1 j}$ columns of the first row of $U^{\prime}$ are ones and the remainder of $U$ coincides with $T$. This proves that $E_{1 n}^{\left(\gamma_{1 n}\right)} \cdots E_{12}^{\left(\gamma_{12}\right)}[T]=[U]$ where the first row of $U$ consists entirely of ones and the remainder of $U^{\prime}$ is identical to $T$. The general result follows by repeating the argument for each of the rows in $T$.

If $V$ is a $U_{K}$-module and $\chi=\left(\chi_{1}, \ldots, \chi_{n}\right)$ an $n$-tuple of non-negative integers, the weight space associated to $\chi$ is the subspace

$$
V^{\chi}=\left\{v \in V: K_{i} v=q^{\chi_{i}} v, 1 \leq i \leq n\right\}
$$

A vector $v \in V^{\chi}$ is a weight vector of weight $\chi$. The weight of a bideterminant $[T]$ in $\nabla_{q}(\lambda)$ is $\chi=\left(\chi_{1}, \ldots, \chi_{n}\right)$ where $\chi_{i}$ is equal to the number of $i$ 's that occur in the tableau $T$. In particular, $\left[T_{\lambda}\right]$ is the unique bideterminant in $\nabla_{q}(\lambda)$ with weight $\lambda$. We will require the following fact, which is easily shown by induction.

Lemma 4.9 Suppose that $[T]$ has weight $\chi=\left(\chi_{1}, \chi_{2}, \ldots, \chi_{n}\right)$. Then $E_{i j}[T]$ belongs to the weight space $\Delta_{q}(\lambda)^{\alpha}$ where $\alpha=\left(\chi_{1}, \ldots, \chi_{i}+1, \ldots, \chi_{j}-1, \ldots, \chi_{n}\right)$.

Theorem 4.10 Suppose that $S$ and $T$ are column-increasing $\lambda$-tableaux and suppose that $T$ is semistandard.

1. If $\Omega_{q}(S, T) \neq 0$ then $[S]$ and $[T]$ have the same weight.

2. If $S$ is semistandard and $\Omega_{q}(S, T) \neq 0$ then $S \leq T$.

Proof: By Lemma 4.9, if $[S]$ and $[T]$ do not have the same weight, $E_{T}$ takes $[S]$ and $[T]$ to different weight spaces. By Theorem $4, E_{T}[T]$ lies in the weight space $\nabla_{q}(\lambda)^{\lambda}$. Thus $\Omega_{q}(S, T)=0$, which completes the proof of 1 .

To prove 2 we assume that $[S]$ and $[T]$ have the same weight. Suppose that $S>T$ and let $t$ be the first entry in the row sequence of $T$ which differs from the corresponding entry $s$ in the row sequence of $S$ so that $s>t$. Let $i$ be the row of the Young diagram in which $t$ occurs in $T$. Suppose that the number of $t$ 's in the $i$ th row of $S$ is equal to $k$ so $\gamma_{i t}>k$. Thus 
$E_{T}[S]=\ldots E_{i t}^{\left(\gamma_{i t}-k\right)} E_{i t}^{(k)} \ldots E_{12}^{\left(\gamma_{12}\right)}[S]$ where $\gamma_{i t}-k>0$. Repeatedly applying Theorem 4.4 we have $E_{i t}^{k} \ldots E_{13}^{\gamma_{13}} E_{12}^{\gamma_{12}}[S]=[k] ! \ldots\left[\gamma_{13}\right] !\left[\gamma_{12}\right] ![U]$ where the first $i-1$ rows of $U$ and the first $\gamma_{i i}+\gamma_{i, i+1}+\cdots+k$ columns of the $i$ th row of $U$ coincide with $T_{\lambda}$ and the remainder of $U$ coincides with $S$. But there are no $t$ 's in the $i$ th row of $U$ and any which appear below the $i$ th are in the first $\gamma_{i i}+\gamma_{i, i+1}+\cdots+k$ columns. Since each of these columns contains an $i$ in a row above such a $t$, we have $E_{i t}^{\gamma_{i t}-k}[U]=0$ so $E_{T}[S]=0$.

Corollary 4.11 The quantum Désarménien matrix is an invertible upper triangular matrix.

The following lemma allows us to deduce a straightening algorithm for quantum bideterminants. For an alternative straightening algorithm, see [11].

Lemma 4.12 Suppose that $S$ and $T$ are column-increasing $\lambda$-tableaux and that $T$ is semistandard. Suppose also that $[S]$ and $[T]$ have the same weight. Then

$$
E_{T}[S]=\Omega_{q}(S, T)\left[T_{\lambda}\right]
$$

Proof: Since $[S]$ and [T] have the same weight, $E_{T}[S]$ and $E_{T}[T]$ both lie in the weight space $\nabla_{q}(\lambda)^{\lambda}$. Thus $E_{T}[S]=c\left[T_{\lambda}\right]=\Omega_{q}(S, T)\left[T_{\lambda}\right]$.

To get a $q$-straightening algorithm, we use the fact that any bideterminant with weight $\chi$ can be expressed as a linear combination of semistandard bideterminants of weight $\chi$ and then call on the Désarménien matrix to give us the coefficients in the decomposition. Let $\Omega_{q, \chi}$ be the submatrix of $\Omega_{q}$ that runs over the $\Omega_{q}(S, T)$ where $[S]$ and $[T]$ have weight $\chi$. Let $\mathcal{I}_{\lambda}^{\chi}=\left\{I \in \mathcal{I}_{\lambda}:\left[T_{I}\right]\right.$ has weight $\left.\chi\right\}$. If $U$ is a column increasing $\lambda$-tableau such that $[U]$ has weight $\chi$, then $[U]=\sum_{I \in \mathcal{I}_{\lambda}^{\chi}} a_{I}\left[T_{I}\right]$. If $J \in \mathcal{I}_{\lambda}^{\chi}$ then

$$
\begin{aligned}
\Omega_{q}\left(U, T_{J}\right)\left[T_{\lambda}\right] & =E_{T_{J}}[U] \text { by Lemma } 4 \\
& =\sum_{I \in \mathcal{I}_{\lambda}^{\chi}} a_{I} E_{T_{J}}\left[T_{I}\right] \\
& =\sum_{I \in \mathcal{I}_{\lambda}^{\chi}} a_{I} \Omega_{q}\left(T_{I}, T_{J}\right)\left[T_{\lambda}\right] .
\end{aligned}
$$

Thus $\left[\Omega_{q}\left(U, T_{I}\right)\right]_{I \in \mathcal{I}_{\lambda}^{\chi}}=\left[a_{I}\right]_{I \in \mathcal{I}_{\lambda}^{\chi}} \Omega_{q, \chi}$ and since $\Omega_{q, \chi}$ is invertible, we have

$$
\left[a_{I}\right]_{I \in \mathcal{I}_{\lambda}^{\chi}}=\left[\Omega_{q}\left(U, T_{I}\right)\right]_{I \in \mathcal{I}_{\lambda}^{\chi}}\left(\Omega_{q, \chi}\right)^{-1} .
$$

Example 4.13 Let $\lambda=(2,1), n=3$, and $\chi=(1,1,1)$. There are two semistandard $\lambda$-tableaux which give bideterminants of weight $\chi$;

$$
T_{1}=\begin{array}{l|l}
1 & 2 \\
\hline 3
\end{array} \quad \text { and } T_{2}=\begin{array}{lll}
\hline 1 & 3 \\
\hline 2
\end{array}
$$


To find $\Omega_{q}\left(T_{1}, T_{2}\right)$, we compute $E_{T_{2}}\left[T_{1}\right]=E_{13}\left[T_{1}\right]=\left(q-q^{-1}\right)\left[T_{\lambda}\right]$. Thus,

$$
\Omega_{q}^{\chi}=\left(\begin{array}{ll}
1 & q-q^{-1} \\
0 & 1
\end{array}\right)
$$

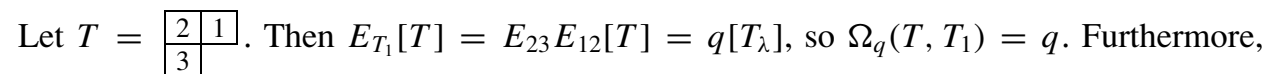
$\Omega_{q}\left(T, T_{2}\right)=-1$. Let $a_{T_{1}}$ and $a_{T_{2}}$ be the coefficients of $\left[T_{1}\right]$ and $\left[T_{2}\right]$ respectively in the straightening decomposition of $[T]$. Then

$$
\left(a_{T_{1}}, a_{T_{2}}\right)=(q,-1)\left(\begin{array}{cc}
1 & q^{-1}-q \\
0 & 1
\end{array}\right)=\left(q,-q^{2}\right), \text { and }[T]=q\left[T_{1}\right]-q^{2}\left[T_{2}\right] .
$$

\section{The connection between two bases of $\Delta_{q}(\lambda)$}

A quantum version of the Carter-Lusztig basis theorem was proved by R. M. Green, [8]. The $q$-Weyl module also has basis dual to the basis of $\nabla_{q}(\lambda)$ in Theorem 3. We would like to discover the relationship between these two bases. We shall prove the standard basis theorem using the quantum Désarménien matrix at the end of this section. Our proof also shows that the standard basis for $\Delta_{q}(\lambda)$ and the basis dual to the basis of semistandard bideterminants for $\nabla_{q}(\lambda)$ are connected by the quantum Désarménien matrix.

We first construct the contravariant dual to $\nabla_{q}(\lambda)$. We begin with a map $\phi: V^{\otimes r} \rightarrow \nabla_{q}(\lambda)$.

Proposition 5.1 The $K$-linear map $\phi: V^{\otimes r} \rightarrow \nabla_{q}(\lambda)$ given by $\phi\left(v_{I}\right)=\left[T_{I}\right]$ is a $U_{K^{-}}$ epimorphism.

Proof: It is clear that $\phi$ is an epimorphism, so we need only prove that $\phi\left(u v_{J}\right)=u\left[T_{J}\right]$ for each $r$-tuple $J$ and each $u \in U_{K}$. Suppose first that $\lambda=\left(1^{r}\right)$ so that $\phi\left(v_{J}\right)=\left[T_{J}\right]$ where $T_{J}$ is a one-column tableau. Let $a$ denote the number of entries equal to $i$ in $T_{J}$. If $a \geq 2$ then $\phi\left(K_{i} v_{J}\right)=q^{a}\left[T_{J}\right]=0=K_{i} \phi\left(v_{J}\right)$. If $a<2$, we also have $\phi\left(K_{i} v_{J}\right)=q^{a}\left[T_{J}\right]=K_{i} \phi\left(v_{J}\right)$.

To prove $\phi\left(E_{i} v_{J}\right)=E_{i} \phi\left(v_{J}\right)$, we may assume that $J$ contains at least one $i+1$, for otherwise equality follows trivially. Suppose first that the number of entries equal to $i+1$ in $J$ is larger than two. Then $E_{i} \phi\left(v_{J}\right)=E_{i}\left[T_{J}\right]=0=\phi\left(E_{i}\left[T_{J}\right]\right)$. Next suppose that the number of entries equal to $i+1$ is less than or equal to two. Then if $J$ also contains an $i$, $E_{i} v_{J}=\sum a_{M} v_{M}$ where each $M$ in the sum has at least two entries equal to $i$, from which it follows that $\phi\left(E_{i} v_{J}\right)=0=E_{i} \phi\left(v_{J}\right)$.

Now suppose that $J$ contains no entries equal to $i$ and one or two entries equal to $i+1$. If $J$ contains exactly one $i+1$, then $E_{i} v_{J}=v_{M}$ where $M$ is identical to $J$ except that the $i+1$ has been replaced with an $i$. Thus, $\phi\left(E_{i} v_{J}\right)=\left[T_{M}\right]=E_{i}\left[T_{J}\right]$. Now suppose that $J$ contains exactly two entries equal to $i+1$ and no entries equal to $i$. Then $E_{i} \phi\left(v_{J}\right)=E_{i}\left[T_{J}\right]=0$. As well, $\phi\left(E_{i} v_{J}\right)=\left[T_{M}\right]+q^{-1}\left[T_{M^{\prime}}\right]$ where the tableaux $T_{M}$ and $T_{M^{\prime}}$ are the same except that an $i$ and an $i+1$ have been interchanged. But $\left[T_{M}\right]+q^{-1}\left[T_{M^{\prime}}\right]=0$ (see [14, Proposition 2.1]) so $\phi\left(E_{i} v_{J}\right)=0$. The proof that $\phi\left(F_{i} v_{J}\right)=F_{i} \phi\left(v_{J}\right)$ is similar. 
For the general case, let $\mu=\left(\mu_{1}, \ldots, \mu_{s}\right)$ be the conjugate of the partition $\lambda=$ $\left(\lambda_{1}, \ldots, \lambda_{k}\right)$. Given $I \in I(n, r)$, let $I=\left(i_{11}, i_{21}, \ldots, i_{\mu_{1} 1}, \ldots, i_{1 s}, \ldots, i_{\mu_{s} s}\right), I_{1}=$ $\left(i_{11}, i_{21}, \ldots, i_{\mu_{1} 1}\right), \ldots, I_{s}=\left(i_{1 s}, i_{2 s}, \ldots, i_{\mu_{s} s}\right)$ so that $v_{I}=v_{I_{1}} \otimes v_{I_{2}} \otimes \cdots \otimes v_{I_{s}}$. The map $\theta: V^{\otimes r} \rightarrow \nabla_{q}\left(\mu_{1}\right) \otimes \nabla_{q}\left(\mu_{2}\right) \otimes \cdots \otimes \nabla_{q}\left(\mu_{s}\right)$ defined by

$$
\theta\left(v_{I_{1}} \otimes v_{I_{2}} \otimes \cdots \otimes v_{I_{s}}\right)=\phi\left(v_{I_{1}}\right) \otimes \phi\left(v_{I_{2}}\right) \otimes \cdots \otimes \phi\left(v_{I_{s}}\right)
$$

is a $U_{K}$-homomorphism. As well, the map $\psi: \nabla_{q}\left(\mu_{1}\right) \otimes \nabla_{q}\left(\mu_{2}\right) \otimes \ldots \otimes \nabla_{q}\left(\mu_{s}\right) \rightarrow \nabla_{q}(\lambda)$ given by $\psi\left(\left[T_{I_{1}}\right] \otimes\left[T_{I_{2}}\right] \otimes \cdots \otimes\left[T_{I_{s}}\right]\right)=\left[T_{I_{1}}\right]\left[T_{I_{2}}\right] \ldots\left[T_{I_{s}}\right]$ is a $U_{K}$-homomorphism. Thus, $\phi=\psi \circ \theta$ is a $U_{K}$-homomorphism as well.

If $V$ and $W$ are two $U_{K}$-modules, a bilinear form $\langle\rangle:, V \times W \rightarrow K$ is said to be $U_{K}$-contravariant if for all $u \in U_{K}, v \in V$, and $w \in W$, we have $\langle u v, w\rangle=\langle v, \tau(u) w\rangle$, where $\tau$ is the antiautomorphism defined in (3.5). We want to define a $U_{K}$-contravariant form on $V^{\otimes r}$ and use it to construct the $q$-Weyl module as is done in the classical case by J. A. Green in [7]. To do so, we introduce the following notation. Given $I \in I(n, r)$, define $\beta(I)$ to be the number of pairs $(a, b)$ for which $a<b$ and $i_{a} \neq i_{b}$. For example, if $I=(1,3,2,1)$ then $\beta(I)=5$. Define a bilinear form $\langle,\rangle_{q}: V^{\otimes r} \times V^{\otimes r} \rightarrow K$ by

$$
\left\langle v_{I}, v_{J}\right\rangle_{q}=q^{\beta(I)} \delta_{I J}
$$

For example, $\left\langle v_{1} \otimes v_{3} \otimes v_{2} \otimes v_{1}, v_{1} \otimes v_{3} \otimes v_{2} \otimes v_{1}\right\rangle=q^{5}$.

Theorem 5.2 The form $\langle,\rangle_{q}: V^{\otimes r} \times V^{\otimes r} \rightarrow K$ is a $U_{K}$-contravariant form.

Proof: Let $I, J \in I(n, r)$ and let $a$ be the number of entries equal to $i$ which occur in $I$ and let $b$ denote the number of entries equal to $i$ which occur in $J$. Then $\left\langle K_{i} v_{I}, v_{J}\right\rangle_{q}=$ $q^{a}\left\langle v_{I}, v_{J}\right\rangle_{q}$ and $\left\langle v_{I}, K_{i} v_{J}\right\rangle_{q}=q^{b}\left\langle v_{I}, v_{J}\right\rangle_{q}$. Both are equal to zero if $I \neq J$ and if $I=J$ then $a=b$ so $\left\langle K_{i} v_{I}, v_{J}\right\rangle_{q}=\left\langle v_{I}, K_{i} v_{J}\right\rangle_{q}$.

Let $I=\left(i_{1}, \ldots, i_{r}\right)$ and $J=\left(j_{1}, \ldots, j_{r}\right)$. Applying (3.1), we have

$$
\begin{aligned}
& F_{i}\left(v_{I}\right)=\left(K_{i, i+1}^{-1} v_{i_{1}}\right) \otimes \cdots \otimes\left(K_{i, i+1}^{-1} v_{i_{r-1}}\right) \otimes\left(F_{i} v_{i_{r}}\right)+\cdots+\left(F_{i} v_{i_{1}}\right) \otimes v_{i_{2}} \otimes \cdots \otimes v_{i_{r}} \text { and } \\
& E_{i}\left(v_{J}\right)=v_{j_{1}} \otimes v_{j_{2}} \otimes \cdots \otimes\left(E_{i} v_{j_{r}}\right)+\cdots+\left(E_{i} v_{j_{1}}\right) \otimes\left(K_{i, i+1} v_{j_{2}}\right) \otimes \ldots \otimes\left(K_{i, i+1} v_{j_{r}}\right) .
\end{aligned}
$$

We will prove that $\left\langle K_{i, i+1}^{-1} v_{i_{1}} \otimes \cdots \otimes K_{i, i+1}^{-1} v_{i_{k-1}} \otimes F_{i} v_{i_{k}} \otimes \cdots \otimes v_{i_{r}}, v_{J}\right\rangle_{q}$ is equal to $\left\langle v_{I}, v_{j_{1}} \otimes \cdots \otimes E_{i} v_{j_{k}} \otimes K_{i, i+1} v_{j_{k+1}} \otimes \cdots \otimes K_{i, i+1} v_{j_{r}}\right\rangle_{q}$ for a fixed $k$ with $1 \leq k \leq r$. We may assume that the $r$-tuples $I$ and $J$ coincide before and after the $k$ th place so we replace all such $i_{l}$ 's in the first form by $j_{l}$ 's. We may also assume that $v_{i_{k}}=v_{i}$ and $v_{j_{k}}=v_{i+1}$. Thus $I=\left(j_{1}, \ldots, j_{k-1}, i, j_{k+1}, \ldots, j_{r}\right), J=\left(j_{1}, \ldots, j_{k-1}, i+1, j_{k+1}, \ldots, j_{r}\right)$. Suppose that there are $a_{1}$ entries equal to $i$ which occur in the $r$-tuple $J$ prior to the $k$ th place and suppose that there are $a_{2}$ entries equal to $i+1$ which occur in $J$ prior to the $k$ th place. Let $b_{1}$ denote the number of entries equal to $i$ which appear in $J$ after the $k$ th place and $b_{2}$ the number of 
entries equal to $i+1$ that appear after the $k$ th place. Then

$$
\begin{aligned}
& \left\langle K_{i, i+1}^{-1} v_{j_{1}} \otimes \cdots \otimes K_{i, i+1}^{-1} v_{j_{k-1}} \otimes F_{i} v_{i} \otimes \cdots \otimes v_{j_{r}}, v_{J}\right\rangle_{q}=q^{-a_{1}} q^{a_{2}} q^{\beta(J)}, \quad \text { and } \\
& \left\langle v_{I}, v_{j_{1}} \otimes \cdots \otimes E_{i} v_{i+1} \otimes K_{i, i+1} v_{j_{k+1}} \otimes \cdots \otimes K_{i, i+1} v_{j_{r}}\right\rangle_{q}=q^{b_{1}} q^{-b_{2}} q^{\beta(I)} .
\end{aligned}
$$

But $q^{-a_{1}} q^{a_{2}} q^{\beta(J)}=q^{-a_{1}} q^{a_{2}} q^{\beta(I)+a_{1}+b_{1}-a_{2}-b_{2}}=q^{b_{1}} q^{-b_{2}} q^{\beta(I)}$ and this completes the proof.

We now take $V_{\lambda, q}$ to be the orthogonal complement to $N=\operatorname{ker} \phi$ with respect to the form $\langle,\rangle_{q}$. It is clear that $V_{\lambda, q}$ is a $U_{K}$-submodule of $V^{\otimes r}$ and the form $(,)_{q}: V_{\lambda, q} \times \nabla_{q}(\lambda) \rightarrow K$ defined by

$$
(x, \phi(y))_{q}=\langle x, y\rangle_{q}, \quad x \in V_{\lambda, q}, y \in V^{\otimes r}
$$

is a non-degenerate contravariant form since $\langle,\rangle_{q}$ is non-degenerate and contravariant and $\phi$ is a $U_{K}$-homomorphism. Thus $V_{\lambda, q} \cong\left(\nabla_{q}(\lambda)\right)^{\circ}$ via the map $\psi: V_{\lambda, q} \rightarrow\left(\nabla_{q}(\lambda)\right)^{\circ}$ defined by

$$
\psi(x)(y)=(x, y)_{q}, \quad x \in V_{\lambda, q}, y \in \nabla_{q}(\lambda) .
$$

One can adjust [7, Proposition 2.7e] appropriately to get a quantum version of that result from which it follows that $V_{\lambda, q} \cong \Delta_{q}(\lambda)$.

The following proof of the standard basis theorem for $\Delta_{q}(\lambda)$ shows that this basis and the basis dual to the basis of Theorem 3 are related by the Désarménien matrix. Recall the definitions of $F_{T}$ and $z_{\lambda}$ from (4.1) and (3.2).

Standard basis theorem-The set $\left\{F_{T} z_{\lambda}: T\right.$ is a semistandard $\lambda$-tableau $\}$ is a $K$-basis for $\Delta_{q}(\lambda)$.

Proof: By definition $z_{\lambda} \in \Delta_{q}(\lambda)$. Let $T_{I}$ and $T_{J}$ be semistandard $\lambda$-tableaux and let $(,)_{q}$ be the non-degenerate contravariant form on $\Delta_{q}(\lambda) \times \nabla_{q}(\lambda)$ defined in (5.1). Then

$$
\left(F_{T_{I}} z_{\lambda},\left[T_{J}\right]\right)_{q}=\left(z_{\lambda}, E_{T_{I}}\left[T_{J}\right]\right)_{q}=\left(z_{\lambda}, \Omega_{q}\left(T_{J}, T_{I}\right)\left[T_{\lambda}\right]+\sum_{M} a_{M}\left[T_{M}\right]\right)_{q}
$$

where each $\left[T_{M}\right]$ in the sum has weight different from $\lambda$. But for each $M$ in the sum, we have $\left(z_{\lambda},\left[T_{M}\right]\right)_{q}=\left\langle z_{\lambda}, v_{M}\right\rangle_{q}=0$. Thus

$$
\left(F_{T_{I}} z_{\lambda},\left[T_{J}\right]\right)_{q}=\left(z_{\lambda}, \Omega_{q}\left(T_{J}, T_{I}\right)\left[T_{\lambda}\right]\right)_{q}=\Omega_{q}\left(T_{J}, T_{I}\right)\left\langle z_{\lambda},\left.v_{J(\lambda)}\right|_{q}=q^{\beta(J(\lambda))} \Omega_{q}\left(T_{J}, T_{I}\right) .\right.
$$

So for each $J \in \mathcal{I}_{\lambda}$ we have $\left(F_{T_{I}} z_{\lambda},\left[T_{J}\right]\right)_{q}=q^{\beta(J(\lambda))} \Omega_{q}\left(T_{J}, T_{I}\right)$. Since $\Omega_{q}=$ $\left[\Omega_{q}\left(T_{J}, T_{I}\right)\right]_{I \in \mathcal{I}_{\lambda}}$ is an upper triangular invertible matrix and the set of semistandard $\lambda$-tableaux forms a $K$-basis for $\nabla_{q}(\lambda)$, the set $\left\{F_{T} z_{\lambda}: T\right.$ is a semistandard $\lambda$-tableau $\}$ is a $K$-basis for $\Delta_{q}(\lambda)$. 


\section{References}

1. R.W. Carter and G.W. Lusztig, "On the modular representations of the general linear and symmetric groups," Math. Z. 136 (1974), 193-242.

2. G. Cliff and A. Stokke, "Codeterminants for the symplectic Schur algebra," J. London Math. Soc, (to appear).

3. J. Désarménien, "An algorithm for the Rota straightening formula," Discrete Math. 30 (1980), 51-68.

4. J. Désarménien, J.P.S. Kung, and G.-C. Rota, "Invariant theory, Young bitableaux, and combinatorics," Adv in Math 27 (1978), 63-92.

5. S. Donkin, The q-Schur Algebra, London Mathematical Society Lecture Note Series 253, Cambridge University Press, Cambridge, 1998

6. J.A. Green, "Combinatorics and the Schur algebra," J. Pure Appl. Algebra 88(1-3) (1993), 89-106.

7. J.A. Green, Polynomial represenations of $G L_{n}$, Lecture Notes in Mathematics 830, Springer 1980 , Berlin/Heidelberg/New York.

8. R.M. Green, " $q$-Schur algebras and quantized enveloping algebras,” Ph.D. thesis, Warwick University, 1995.

9. J. Hu, "A combinatorial approach to representations of quantum linear groups," Comm. Algebra 26(8) (1998), 2591-2621.

10. R.Q. Huang and J.J. Zhang, "Standard basis theorem for quantum linear groups," Adv. Math. 102 (1993), 202-229.

11. B. Leclerc and J. Thibon, "The Robinson-Schensted correspondence, crystal bases, and the quantum straightening at $q=0$," Electron. J. Combin. 3(2) (1996), R11.

12. G. Lusztig, "Finite dimensional Hopf algebras arising from quantized universal enveloping algebras," J. Am. Math. Soc. 3 (1990), 257-297.

13. A. Stokke, "A symplectic Désarménien matrix and a basis for the symplectic Weyl module," J. Algebra 272(2) (2004), 512-529.

14. E. Taft and J. Towber, "Quantum deformation of flag schemes and Grassmann schemes I-A $q$-deformation of the shape algebra for $G L(n)$, , J. Algebra 142 (1991), 1-36.

15. M. Takeuchi, "Some topics on $G L_{q}(n)$, , J. Algebra 147 (1992), 379-410. 